\title{
Disuria: consigli pratici di gestione nei pazienti eleggibili alle cure palliative pediatriche
}

\author{
Irene Avagnina, Letizia Bertasi, Franca Benini \\ Centro Regionale Veneto di Terapia del Dolore e Cure Palliative Pediatriche, Dipartimento di Salute della Donna \\ e del Bambino, Università di Padova
}

\section{Introduzione}

Le cure palliative pediatriche (CPP) rappresentano un ambito multidisciplinare della pediatria che si prefigge l'obiettivo di garantire la qualità di vita a bambini affetti da malattie inguaribili ad alta complessità assistenziale e di rispondere ai loro bisogni e a quelli delle loro famiglie.

A livello clinico, molteplici sono i sintomi disturbanti con ampia ricaduta sulla qualità della vita, che devono essere gestiti $\mathrm{e}$ trattati. Alcuni di questi in modo particolare rappresentano una sfida importante per i caregiver e i sanitari che si occupano dei bambini eleggibili alle CPP.

In questo lavoro e nei successivi verranno affrontati sintomi legati all'apparato genitourinario e gastroenterologico che spesso riscontriamo in bambini in CPP.

\section{Definizione e eziologia}

La disuria si definisce come una minzione dolorosa e può essere secondaria a una contrazione dolorosa della muscolatura della vescica o al contatto dell'urina con la mucosa genitourinaria infiammata [1]. Dati epidemiologici sulla disuria in età pediatrica non sono disponibili e vanno ricercati piuttosto sulla incidenza delle specifiche cause tra cui sicuramente le più studiate sono le infezioni urinarie.

Nell'ambito delle cure palliative pediatriche la disuria può essere difficilmente riconoscibile oltre che nei lattanti anche nei bambini con compromissione neurologica per i quali nel percorso clinico di inquadramento del dolore non vanno dimenticate le cause nefrourologiche.

Le cause di disuria, in ambito pediatrico, sono molteplici e, per facilitarne l'inquadramento, le suddivideremo in due macroaree: infettive e non infettive [2-3] (Box 1). Altri fattori influenzano l'eziopatogenesi della disuria come l'età, il sesso, la presenza di patologie e/o comorbidità (malformazioni nefrourologiche, patologie metaboliche, disfunzioni minzionali, disidratazione, nutrizione enterale, immobilità), alcune terapie farmacologiche; pertanto nell'inquadramento diagnostico del sin-

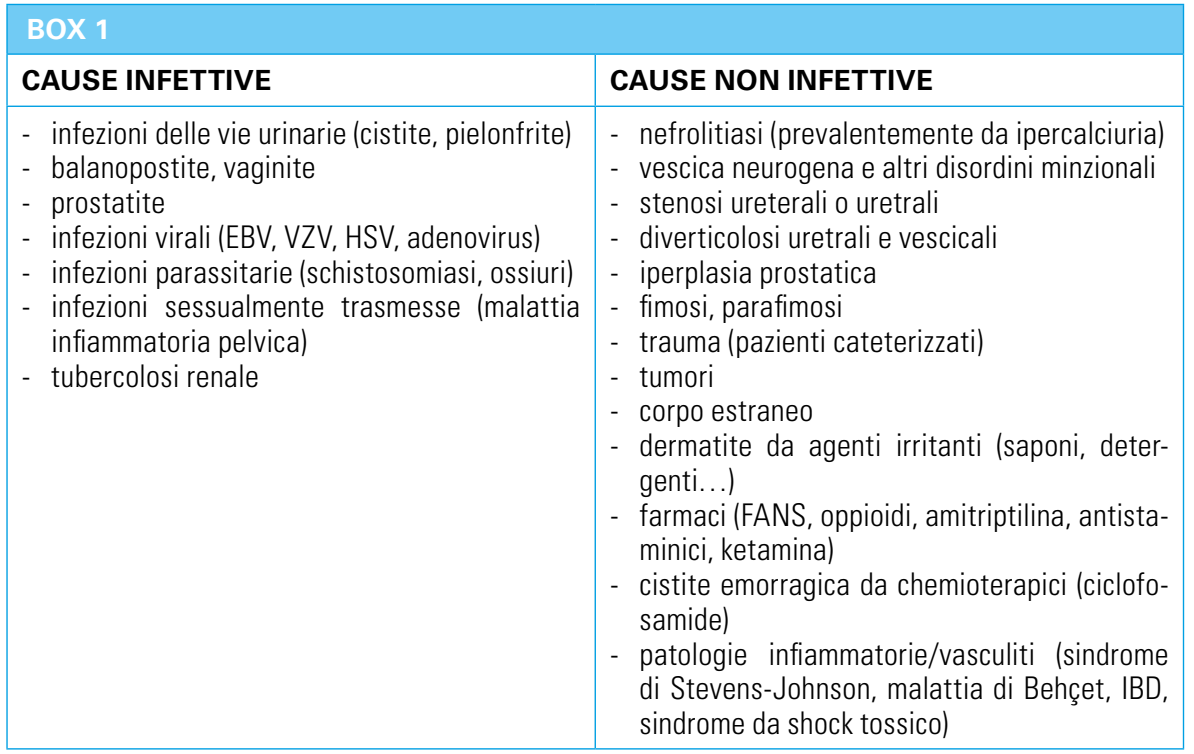

tomo è necessaria una valutazione globale del bambino.

I bambini in cure palliative hanno una maggior predisposizione a presentare disuria rispetto alla popolazione generale in quanto spesso presentano quei fattori predisponenti sovramenzionati. Tuttavia, mancano dati di incidenza in questa popolazione.

In base alla nostra esperienza, in CPP, le cause di disuria più frequenti sono quelle di natura infettiva, i disturbi minzionali su base neurologica, la nefrolitiasi, i traumi e una possibile correlazione con terapie farmacologiche (oppioidi, antiepilettici). Inoltre, i bambini con vescica neurogena che vengono sottoposti a cateterismo estemporaneo o i bambini con cateteri vescicali a permanenza sono a maggior rischio per infezioni e traumatismi. Non vanno poi misconosciute le cause di origine genitale, come vaginiti e prostatiti, che possono essere osservate soprattutto nell'adolescente e giovane adulto.

\section{Sintomatologia}

Le manifestazioni cliniche più frequentemente associate alla disuria sono:

- anomalie minzionali: ritenzione urinaria (da disfunzione minzionale o come meccanismo di difesa per evitare il dolore), oliguria, pollachiuria;
- anomalia delle caratteristiche delle urine: piuria, ematuria, urine maleodoranti, renella;

- segni locali: dermatite, iperemia cutanea, tumefazione, edema, lesioni ulcerose, ferite/ecchimosi, presenza di secrezioni purulente, sanguinamento $\mathrm{cu}^{-}$ taneo;

- sintomi sistemici: febbre, rash o altre lesioni cutanee generalizzate, malessere, perdita di peso.

Il dolore può essere correlato esclusivamente alla minzione o può essere presente un dolore genitourinario persistente che viene peggiorato dalle minzioni. Inoltre alla disuria può essere associato un dolore in regione pelvica (i.e. per la prostatite nel maschio) o un dolore addominale (i.e. per la pielonefrite e la nefrolitiasi).

\section{Diagnosi}

Il percorso diagnostico in caso di disuria si avvale della valutazione clinica e di specifici esami laboratoristici e strumentali.

\section{Anamnesi}

A livello anamnestico va indagata la frequenza degli episodi di disuria, da quanto è insorto il sintomo, le abitudini minzionali (numero di minzioni, se il panno 


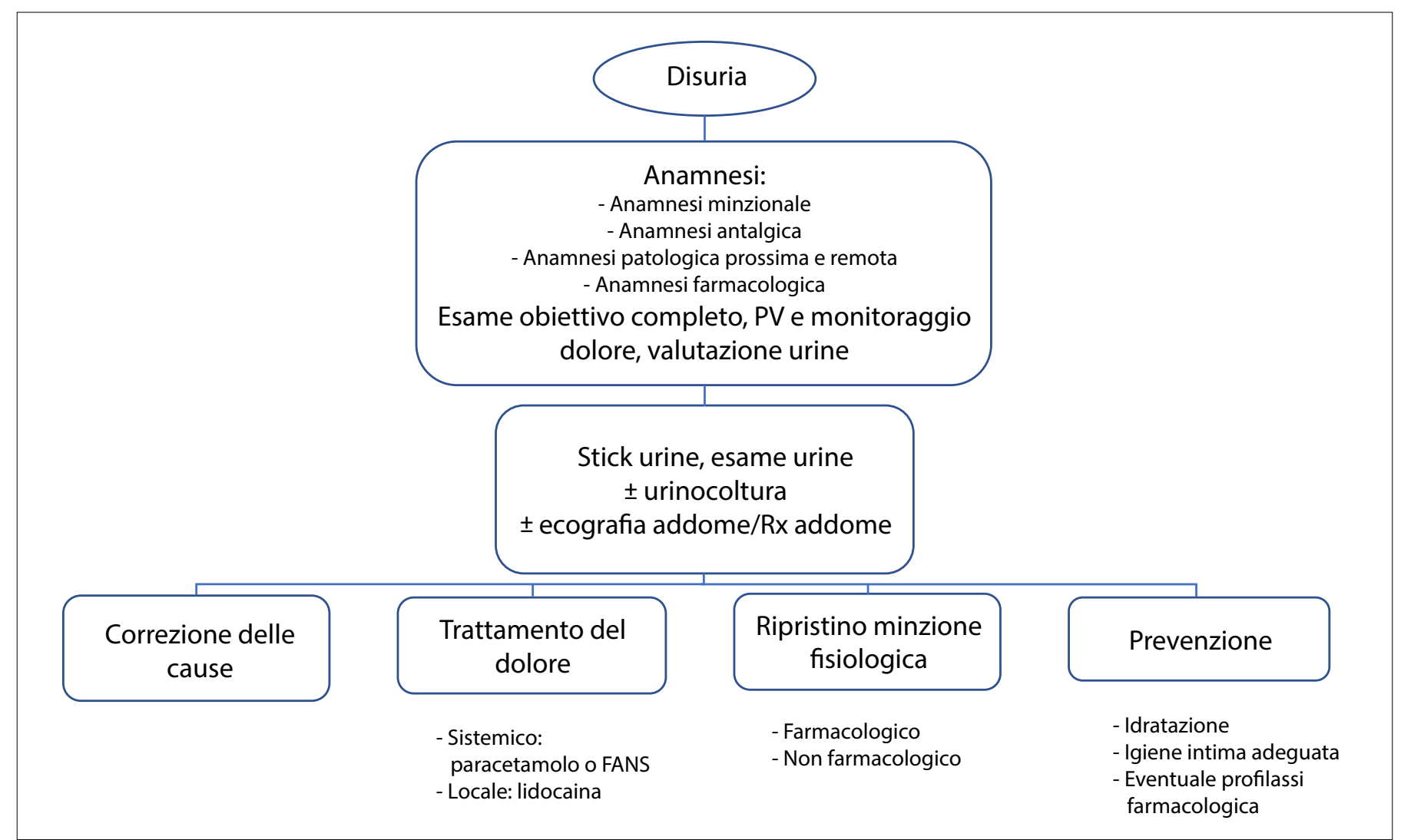

Figura 1. Flow chart per la gestione della disuria in CPP.

è pieno o no, se viene osservato gocciolamento interminzionale) e le eventuali modifiche osservate dall'insorgenza della disuria, le caratteristiche delle urine (aspetto, colore, odore) e delle secrezioni genitali (colore, aspetto, consistenza; per esempio sono biancastre/caseiformi nella vaginite da Candida e scarse e incolori nella vaginite da Chlamydia o da flora saprofita). Vanno ricercati segni o sintomi correlati inquadrabili in un'eziologia infettiva/infiammatoria (febbre, rash o lesioni cutanee, sintomi gastroenterologici, artrite, congiuntivite/uveite ecc.) o oncologica (calo ponderale, astenia), storia di trauma, storia di infezioni urinarie ricorrenti, lo sviluppo puberale, le abitudini di igiene intima (uso di detergenti, frequenza e modalità di igiene intimo) e le abitudini alimentari, l'anamnesi farmacologica.

Il clinico deve conoscere e ricercare i fattori di rischio principali per le cause di disuria. Riportiamo di seguito quelli per le infezioni urinarie e la nefrolitiasi, che sono tra le cause più frequentemente riscontrabili in CPP.

Fattori predisponenti le infezioni urinarie: vescica neurogena, necessità di cateterizzazioni, disidratazione, stipsi, malformazioni nefro-urologiche, paziente immunocompromesso, storia di pregresse infezioni.

Fattori predisponenti lo sviluppo di nefrolitiasi [4-5]: situazioni che determinano ipercalciuria (i.e. ipervitaminosi D, iper- paratiroidismo, aumentato rimodellamento osseo con bassa densità ossea), disidratazione, immobilità (pazienti con impossibilità deambulazione), farmaci (cortisonici per il rimodellamento osseo, allopurinolo, antiepilettici come il topiramato), diete iperproteiche, dieta chetogenica, alimentazione enterale, patologie di base metaboliche, fibrosi cistica, sindromi genetiche e malformazioni nefrourologiche, infezioni urinarie ricorrenti.

Per quanto riguarda la valutazione del dolore è fondamentale approfondire la valutazione antalgica indagando da quanto è insorto il dolore, la sua intensità, il suo andamento nel tempo, l'irradiazione o la presenza di altre sedi di dolore, la presenza di fattori scatenanti e/o attenuanti, la risposta a determinate classi di antidolorifici. A ciò va affiancata un'adeguata misurazione del dolore con scale algometriche validate per età $\mathrm{e}$ patologia di base.

\section{Esame obiettivo}

La valutazione clinica prevede l'esame obiettivo completo con particolare attenzione alla regione genitale e addominale. Vanno visionate eventuali lesioni, rash o dermatiti. Vanno visionate le urine. È utile rilevare i parametri vitali completi di pressione e temperatura e i parametri antropometrici. Infine, vanno ricercate ed esaminate le zone dolenti.
Accertamenti laboratoristici e strumentali In pazienti che presentino disuria è fondamentale raccogliere un campione di urina il più sterilmente possibile per l'esame urine con sedimento e lo stick urine. Tali accertamenti possono aiutare il clinico a valutare la presenza di segni di infezione (principalmente leucocituria, batteri nel sedimento e nitriti nelle urine); segni di disidratazione (alto peso specifico, chetonuria); presenza di microematuria, proteinuria, alterazioni del $\mathrm{pH}$ urinario e presenza di cristalli monoamorfi ed emazie al sedimento.

In caso di riscontro di segni di infezione all'esame urine la diagnosi deve essere confermata tramite urocoltura, con campione urine che andrà raccolto sempre il più sterilmente possibile.

Nel sospetto di renella o micronefrolitiasi utile la ricerca su due campioni estemopanei di elettroliti urinari (calcio, fosforo, magnesio, acido urico, citrato e creatinina urinaria) e l'esecuzione di ecografia addome e/o rx addome.

In caso di infezioni ricorrenti o riscontro di calcolosi renale utile un consulto con nefrologo o urologo pediatra per definire successiva strategia diagnostico-terapeutica.

\section{Trattamento}

Il trattamento della disuria si basa essenzialmente sulla correzione della causa specifica (per le quali si rimanda alle linee gui- 
da di patologia e/o a consultazione con nefrourologo pediatra) e sul ripristino di adeguata fisiologia minzionale (argomento che verrà trattato nella rassegna sulla vescica neurogena). L'uso di terapia antidolorifica per via orale (i.e. paracetamolo e/o FANS) associato ad anestetici topici (i.e. lidocaina pomata) possono favorire a ridurre il dolore esacerbato dalla minzione e conseguentemente a facilitare la minzione stessa.

Di seguito riportiamo alcune strategie terapeutiche che possono aiutare i clinici nella prevenzione di alcune cause di disuria:

- in caso di pregressi episodi di nefrolitiasi, segni di microlitiasi o/e in presenza di fattori di rischio per nefrolitiasi considerare profilassi con citrato di potassio (Citrak ${ }^{\circledR}$ una bustina/die) in caso di ipercalciuria (con monitoraggio successivo di EGA/potassio/pH urinario) $[6]$;

- in caso di infezioni ricorrenti delle basse vie urinarie considerare nelle femmine profilassi con mirtillo rosso (i.e. $\mathrm{Ci}^{-}$ stonorm ${ }^{\circledR}$ una o mezza bustina die) ed eventuale profilassi antibiotica in base al patogeno identificato;

- regole di igiene intima: evitare un uso eccessivo di detergenti intimi. Utilizzo di pomate topiche in caso di dermatiti o lesioni genitali;

- regole nutrizionali: garantire adeguati apporti di acqua anche in pazienti alimentati per via enterale, garantire i fabbisogni di calcio e vitamina $\mathrm{D}$.

Infine, un aspetto importante in CPP è l'associazione tra disuria e uso di oppioidi. La prevalenza di disuria nei pazienti adulti oncologici che assumono oppioidi è di circa il $14,9 \%$, con incremento della sintomatologia all'aumentare della dose dell'op- pioide [7]. La maggior parte degli oppioidi riducono il tono simpatico incrementando l'azione parasimpatica, modificando fattori urodinamici.

Il trattamento della disuria da oppioidi non è ancora stato codificato. L'utilizzo di flavossato-propifenazone (Cistalgan ${ }^{\circledR}$ ), usato come trattamento sintomatico sembra relativamente efficace, nonostante siano necessari ancora ulteriori studi [8].

\section{Take home message}

- La disuria può essere un sintomo doloroso misconosciuto in pazienti con compromissione neurologica.

- Oltre alle infezioni delle vie urinarie altre cause di disuria vanno escluse, tra le quali particolare attenzione va posta alle calcolosi renali.

- Il trattamento si basa sulla prevenzione e sulla correzione delle cause.

\section{Caso clinico}

Ragazzo di 18 anni con esiti neurologici da infezione perinatale da Listeria monocytogenes, peso $24 \mathrm{~kg}$. Storia di disuria e dolore pelvico associato a riscontro di secrezioni genitali purulente e rosate da circa un mese.

A livello anamnestico si segnala: paziente con tendenza alla ritenzione urinaria necessitante in alcune occasioni di cateterismi estemporanei, postura seduta obbligata con pressione costante a livello pelvico. Descrive un dolore urente interno a livello pelvico che causa anche risvegli notturni, disuria con riduzione delle minzioni spontanee. Riferita dal caregiver impressione di dolore e difficoltà all'esecuzione del cateterismo. Obiettivamente: esame obiettivo addominale e pelvico nella norma. Non alterazione dei parametri vitali.
Urine: urine torpide, presenza di renella, maleodoranti. Confermate secrezioni roseo-purulente anche extraminzionali.

Esami di laboratorio: indici di flogosi negativi, funzionalità renale conservata. Esame urine con riscontro di cistalli amorfi e numerosi batteri al sedimento. Urinocoltura positiva per Enterococco faecium.

Eseguita consulenza urologica: dato il quadro clinico diagnosi di prostatite per cui impostata terapia antibiotica con ciprofloxacina per tre settimane con risoluzione.

Per la gestione antalgica impostata una terapia fissa con ibuprofene $10 \mathrm{mg} / \mathrm{kg} \times 3 \mathrm{e}$ novalgina $10 \mathrm{mg} / \mathrm{kg} \times 3$ per la prima settimana, poi progressivamente scalata in base all'andamento del dolore.

irene.avagnina@aopd.veneto.it

1. Kliegman RM. Pediatric Symptoms-Based Diagnosis. Nelson, 2018.

2. Fleisher GR, Teach SJ, Duryea TK, Wiley JF. Etiology and evaluation of dysuria in children and adolescents. Uptodate, 2019

3. Mehta A, Williams V, Parajuli B. Child with Dysuria and/or Hematuria. Indian J Pediatr. 2017 Oct;84(10):792-798.

4. Acar B, Inci Arikan F, Emeksiz S, Dallar Y. Risk factors for nephrolithiasis in children. World J Urol. 2008 Dec;26(6):627-630.

5. Reusz GS, Hosszu A, Kis E. Evaluation of a child with suspected nephrolithiasis. Curr Opin Pediatr. 2020 Apr;32(2):265-272.

6. Edefonti A. Manuale di nefrologia Pediatrica. Esculapio, 2015.

7. Mercadante S, Ferrera P, Casuccio A. Prevalence of opioid-related dysuria in patients with advanced cancer having pain. Am J Hosp Palliat Care. 2011 Feb;28(1):27-30.

8. Mercadante S. Opioid Analgesics Adverse Effects: The Other Side of the Coin. Curr Pharm Des. 2019;25(30):3197-3202. 